\title{
Comparison of Procedures to Evaluate the Cyclic Stress-Strain Curve from Incremental Step Test
}

\author{
Giovanni Zonfrillo and Duccio Nappini \\ Department of Industrial Engineering, University of Florence, Florence 50139, Italy
}

\begin{abstract}
The design of structural components in low-cycle fatigue field often requires the knowledge of the cyclic properties of the material, which are commonly described by the classical relation of Ramberg-Osgood. In order to obtain the cyclic curve using experimental data from incremental step tests, four methodologies are described and critically discussed. Three methods differ in the procedure of evaluation of the elastic modulus, while in the last one the experimental data are interpolated with a single non-linear regression. The various techniques were applied to data obtained from tests carried out on stainless steel specimens, and the resulting differences were analysed and quantified. An average behaviour was evaluated considering the total set of data obtained from experimental tests. The choice of the most suitable method is related to both the strain range of interest and the goal for which the results are used.
\end{abstract}

Key words: Cyclic curve, incremental step test, Ramberg-Osgood, stainless steel.

\section{Introduction}

During service, structural components can be subject to stress that exceeds the elastic limit, especially when local stress concentration is involved. For these applications, fatigue design requires a special attention to the evaluation of stress and strain fields in the critical areas. The stress-strain responses of many materials change significantly when elasto-plastic cyclic loading conditions are applied, and therefore, it is necessary to refer to stable conditions described by cyclic stress-strain curve. From the analytical point of view, the cyclic curve can be expressed in the classic form of a Ramberg-Osgood relation:

$$
\varepsilon_{a}=\frac{\sigma_{a}}{E}+\left(\frac{\sigma_{a}}{K^{\prime}}\right)^{1 / n^{\prime}}
$$

where, $\sigma_{a}$ and $\varepsilon_{a}$ are the stress and the strain amplitude, respectively, $E$ is the elastic modulus, $K^{\prime}$ is the cyclic strength coefficient and $n^{\prime}$ is the cyclic strain hardening exponent [1]. Admitting the Masing hypothesis, this relationship is used to describe the

Corresponding author: Giovanni Zonfrillo, associate professor, Ph.D., research field: behaviour of materials, fatigue, life prediction.E-mail : giovanni.zonfrillo@unifi.it. hysteresis loops of metals and alloys and, in conjunction with Neuber's rule, to evaluate the local notch tip stresses and strains values [2]. A practical drawback of Eq. (1) is that the relation is not analytically invertible and it is necessary to obtain a solution of this problem using numerical procedures or approximate expressions [3].

Therefore, the cyclic curve is characterized by the values of the three material coefficients: $K^{\prime}, n^{\prime}$ and $E$. In literature, various approaches are presented in order to estimate the materials cyclic properties from experimental monotonous data, using analytical formulations [4-6] or neural network interpolation methodologies [7, 8]. Unfortunately, these formulations have a statistical nature and should be applied only to material classes (usually steel) used for their validation. From a theoretical point of view, it is possible to estimate $K^{\prime}$ and $n^{\prime}$ from compatibility equation [9] using oligocyclic fatigue data, but this approach leads to a roughly evaluation of true values, with a significant discordance respect to experimental results. Numerous correlations between these parameters and in particular between the exponents were proposed in other studies $[10,11]$. 
In order to perform a correct evaluation of the constants in Eq. (1), a set of dedicated experimental tests should be carried out. The three commonly used methods are: companion (single step), multiple step and incremental step test $[12,13]$. A discussion about the difference between the results obtained from these testing procedures is reported in $[14,15]$.

In the present work, four methodologies are described and critically discussed in order to obtain the cyclic curve from the results of incremental step tests. The various techniques were applied to data obtained from tests carried out on stainless steel specimens, and the resulting differences were analysed and quantified. The paper is organized as follows: Section 2 explains the experimental apparatus and the procedure; Section 3 introduces the data analysis methodologies; Section 4 presents experimental results and the relative discussion; finally Section 5 gives conclusions.

\section{Methods and Procedures}

Incremental step tests were carried out on $\mathrm{X} 22 \mathrm{CrMoV} 12-1$ steel at room temperature. Repeated loading blocks were imposed on the specimen until the stabilization of the hysteresis loops.

Samples were machined according to the ASTM E606-04 specification and tested in strain control at room temperature on a digital closed-loop servo-hydraulic test machine; the strain was measured by an extensometer with the gage length of $12 \mathrm{~mm}$. In each block of cycles, the strain amplitude firstly increases until a predetermined maximum strain value and after decrease until zero. The lines connecting the tips of the strain cycles form a rhombus (Fig. 1).

The applied set of cycles was performed at a constant strain rate of $0.002 \mathrm{~s}^{-1}$; each block consists of fifty symmetrical cycles and starts at zero strain; the maximum strain value of first cycle in the block is equal to 0.001 . Starting from these assumptions, the block is fully defined by imposing the designated maximum strain amplitude. Tests were stopped after stabilization, usually after the application of 5 or 6 blocks.

Actuator displacement, time, load and total strain signals were acquired (2,000 points per channel) during the tests. Fig. 2 portrays an example of hysteresis loops consequent to the application of a block of cycles. To evaluate the dispersion of the results, five samples were tested in identical conditions, with a maximum strain value of 0.01 .

\section{Data Analysis}

Various methodologies were applied to experimental data in order to obtain the cyclic stress-strain curve of the material. While the monotonous stress-strain curve could be obtained directly from recorded data, the cyclic stress-strain curve needs a suitable post process. The first step consists in the determination of the stress and strain amplitude values for each hysteresis

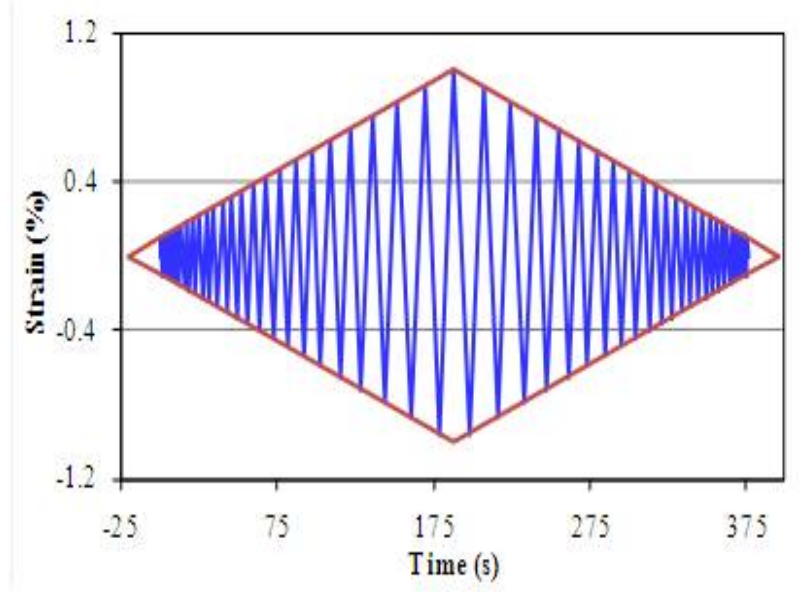

Fig. 1 Imposed strain in function of time.

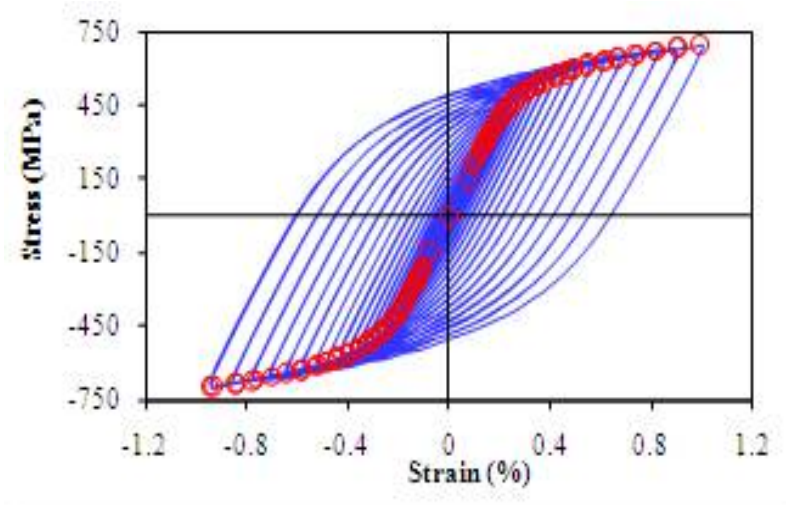

Fig. 2 Hysteresis loops in a block of cycles and their peaks. 
loop in the block. These values, plotted in a stress-strain diagram, represent the data which must be interpolated to obtain the analytic expression of the cyclic curve (referred to the chosen block of cycles), as shown in Fig. 2.

The determination of the three parameters $E, K^{\prime}, n$, could be performed with two different approaches.

The first approach needs firstly the determination of Young's modulus to decompose the total strain in the elastic and plastic components; the remaining two parameters could be assessed with a linear regression of $\sigma_{a}$ versus plastic strain amplitude in the bi-logarithmic scale. It is clear that to obtain a reliable prediction of the material behaviour an accurate estimation of Young's modulus is required. The alternative approach requires a complex numerical procedure: the three values which involve the best fit of the experimental data are evaluated at the same time by means of a non-linear regression analysis. In this case, the Young's modulus is the "cyclic" value of the parameter.

\subsection{Method 1}

In order to estimate the Young's modulus three different procedures have been applied, namely $1 \mathrm{a}, 1 \mathrm{~b}$ and $1 \mathrm{c}$.

\subsubsection{Procedure 1a}

Only the tips of the stable elastic hysteresis loops are considered and the elastic modulus is estimated as the angular coefficient of the best fitting straight line using least squares regression analysis. The data in the elastic field are identified by an iterative procedure:

- The best fit line for a increasingly number of data starting from zero is estimated;

- The value of the Euclidean norm of the regression residuals is calculated;

- The largest set of data for which the Euclidean norm is lower than a threshold value, assumed equal to $10 \mathrm{MPa}$, is determined.

\subsubsection{Procedure $1 \mathrm{~b}$}

The values of the unloading modulus (both in tension and in compression) for each hysteresis loop are estimated and an arithmetic mean is carried out. The range of values on which the linear regression is performed is based on two parameters, expressed as percentage of the stress and strain peak values [16]; in detail, the range considered starts from a point for which the strain varies $2 \%$ compared to the peak value and it has an amplitude set to $60 \%$ of peak stress.

\subsubsection{Procedure 1c}

This methodology requires all the experimental points recorded in the terminal part of the block (in particular in the last 6 cycles), surely in the elastic region. The value of Young's modulus is obtained as the angular coefficient of the straight line that better interpolate these data set.

3.1.4 Evaluation of the Points in the Plastic Region

Once estimated the elastic modulus value, it is necessary to determine the points belonging to the plastic strain field in order to perform a linear regression in bi-logarithmic scale and to obtain the n' and $\mathrm{K}$ ' values.

From a theoretical point of view would be enough to identify the first point (with the lowest stress value) for which the plastic strain is greater than zero. Unfortunately, in the elastic field the acquired experimental values deviate a bit from the straight line that describe their trend, since an interpolation error occurs. To obtain the first point inside the plastic range, five consecutive recorded points must be overcome, on average, a positive threshold value of plastic strain, assumed equal to 0.01 .

\subsection{Method 2}

In this method, the three characteristic material parameters of the analytic expression of the cyclic curve are evaluated at the same time using a least squares root method. The Ramberg Osgood relationship is the nonlinear model to be used. Therefore, the dependent variable $\varepsilon_{a}$ is function of both the independent variable $\sigma_{\mathrm{a}}$ and the three parameters $E$, $n^{\prime}, K^{\prime}: \varepsilon_{a}=f\left(\sigma_{a}, E, n^{\prime}, K^{\prime}\right)$. 
With reference to a generic experimental point with coordinates $\left(\sigma_{a i}, \varepsilon_{a i}\right)$, the relationship $S_{i}=\left[\varepsilon_{a i}-f\left(\sigma_{a i}, E\right.\right.$, $\left.\left.n^{\prime}, K^{\prime}\right)\right]^{2}$ defines the square of the deviation between the measured strain at $i$-th point and the value assumed by the function $f$ when $\sigma_{a}=\sigma_{a i}$. The cyclic parameters values will be those which minimize the sum of $S_{i}$, evaluated on the whole set of points.

Whereas the linear regression model performs a closed-form solution, this method requires an iterative procedure to converge to a solution. For this purpose, was developed a Matlab $^{\odot}$ code which is able to estimate the material coefficients $E, n^{\prime}, K^{\prime}$, starting from the values obtained by the procedure $1 \mathrm{c}$. In order to ensure the reliability of the solution, it was verified that the result obtained was not a function of starting values. Indeed starting values affect only the number of iterations to obtain the convergence.

\section{Application to X22CrMoV12-1 Stainless Steel}

The methodologies were applied to data obtained from tests carried out on X22CrMoV12-1 stainless steel. The alloy showed cyclic softening.

\subsection{Young's Modulus}

The Young's modulus values, calculated with the various procedures, are shown in Table 1.

The scatter of data (related to the average value) obtained on five samples, regardless of the applied procedure, shows a mean values less than $2.5 \%$, with the highest value related to method 2 .

The analysis of the results obtained on each sample, as the method changes, shows values of dispersion slightly higher; approach 1a gives on average lowest values, instead the highest values are given by approach 2 .

However, it should be noted that the value of $E$ obtained with procedure 2 is not the true value of material Young's modulus; in this case, $E$ is only one of three variables suitable to describe the material behaviour. The Ramberg-Osgood relation satisfactorily
Table 1 Values of modulus of elasticity evaluated with various methods.

\begin{tabular}{lllll}
\hline \multirow{2}{*}{ Specimen } & \multicolumn{4}{c}{ Modulus of elasticity (GPa) } \\
\cline { 2 - 5 } & Method 1a & Method 1b & Method 1c & Method 2 \\
\hline 1 & 197.8 & 210.1 & 207.9 & 210.8 \\
2 & 202.6 & 214.7 & 212.9 & 214.4 \\
3 & 200.8 & 209.2 & 209.1 & 214.3 \\
4 & 208.8 & 206.8 & 208.7 & 210.2 \\
5 & 199.7 & 214.5 & 213.8 & 228.8 \\
\hline
\end{tabular}

describes the plastic part of the curve $\varepsilon_{a}-\sigma_{a}$, but it does not consider an initial linear behaviour, because the plastic component of the strain is always present for any value of $\sigma_{a}$.

The value of the elastic modulus obtained with the method 1a may be significantly different from the one evaluated in a tensile test [16]. Moreover, an additional difficulty is due by the fact that the points used for the regression analysis may be insufficient to an accurate estimation, since they depend on the number of cycles inside the imposed load block.

The evaluation performed with procedure $1 \mathrm{~b}$ could be present a variability factor due to the possible difference in the traction-compression material behaviour. If the values achieved in the two different phases are different, the cyclic curve evaluation should be carried out separately for traction and compression stresses. In the case studied the difference is of the order of $3 \%$, with highest value in traction.

\subsection{Cyclic Strain Hardening Exponent and Strength Coefficient}

The values of the parameters obtained for the plastic component of strain amplitude are shown in Table 2.

It is observed a greater dispersion than the one obtained for the Young's modulus, especially as regards the value of $K$.

Again, the scatter among the results obtained applying the four methodologies to a single sample are higher than that obtained using the same methodology on the various samples.

On the other hand, these considerations valid from a mathematical point of view, are not directly linked to 
Table 2 Values of $n^{\prime}$ and $K^{\prime}$ evaluated with various methods.

\begin{tabular}{|c|c|c|c|c|c|c|c|c|}
\hline \multirow{3}{*}{ Sample } & \multicolumn{2}{|c|}{ Method 1a } & \multicolumn{2}{|c|}{ Method $1 b$} & \multicolumn{2}{|c|}{ Method 1c } & \multicolumn{2}{|c|}{ Method 2} \\
\hline & $K^{\prime}$ & $n^{\prime}$ & $K^{\prime}$ & $n^{\prime}$ & $K^{\prime}$ & $n^{\prime}$ & $K^{\prime}$ & $n^{\prime}$ \\
\hline & $\mathrm{MPa}$ & & $\mathrm{MPa}$ & & $\mathrm{MPa}$ & & $\mathrm{MPa}$ & \\
\hline 1 & 1239 & 0.1100 & 1594 & 0.1506 & 1498 & 0.1407 & 1613 & 0.1371 \\
\hline 2 & 1152 & 0.1028 & 1444 & 0.1392 & 1411 & 0.1353 & 1432 & 0.1379 \\
\hline 3 & 1274 & 0.1197 & 1410 & 0.1376 & 1408 & 0.1373 & 1518 & 0.1505 \\
\hline 4 & 1358 & 0.1323 & 1351 & 0.1301 & 1354 & 0.1318 & 1407 & 0.1382 \\
\hline 5 & 1157 & 0.1034 & 1398 & 0.1361 & 1370 & 0.1328 & 1673 & 0.1680 \\
\hline
\end{tabular}

the reliability of the material behaviour evaluation, since it is important to evaluate the dispersion in the cyclic curve, given by the set of the three parameters, and not the scatter of the single coefficients.

\subsection{Dispersion of Results}

Different values for $E, n^{\prime}$ and $K$ ' could lead to cyclic curves that from an engineering point of view are sufficiently approximate; in order to estimate the data dispersion, it is useful to calculate the differences in a well-defined strain range. It is necessary to set a maximum threshold value, because increasing the strain amplitude the cyclic curves tend to diverge progressively from each other. The threshold value was chosen equal to 0.02 , because for highest values the life of a component would be only few cycles and so not interesting for engineering applications.

To perform the comparison, it is necessary to define a suitable parameter proportional to the deviation between the various curves, so as to be able to carry out a quantitative evaluation of the dispersion.

An obvious choice would be to determine the stress value obtained in the various cases for the same strain; extending this parameter to a range, the area under the curves evaluated in the strain range considered was used. As a first step the cyclic curves, obtained by applying the same methodology to the five tested samples are compared. Fig. 3 shows for example the curves obtained with the procedure 2; similar representations are obtained also with the other methodologies. The highest differences, as expected, occur essentially in the plastic field. In order to quantify these differences, the values of the area under the curves in the range $0<\varepsilon_{a}<0.02$ are reported in Fig. 4. Table 3 shows the maximum, minimum and mean values of the difference between these areas, estimated on each sample. These data are related to the dispersion of results attributable to the material.

\subsection{Methodologies Comparison}

Fig. 5 (related to sample 1) shows a comparison

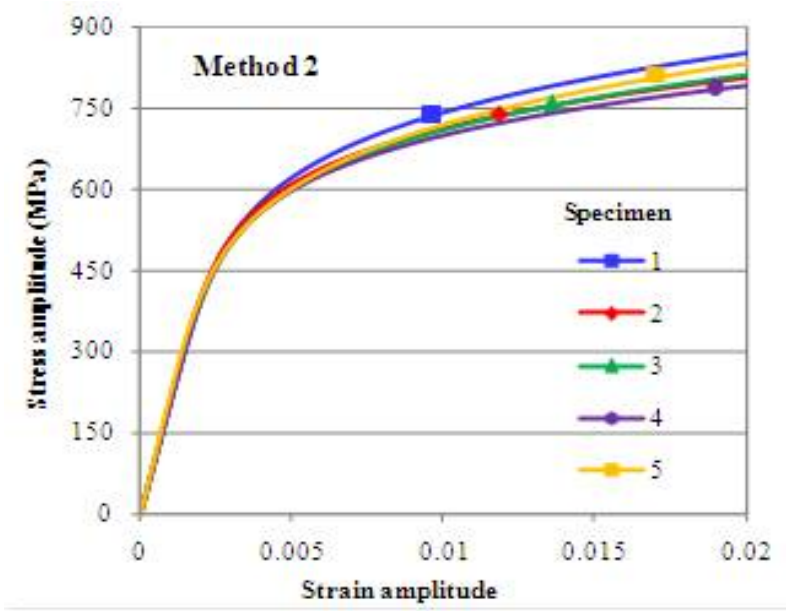

Fig. 3 Cyclic curves obtained by applying the method 2 to various specimens.

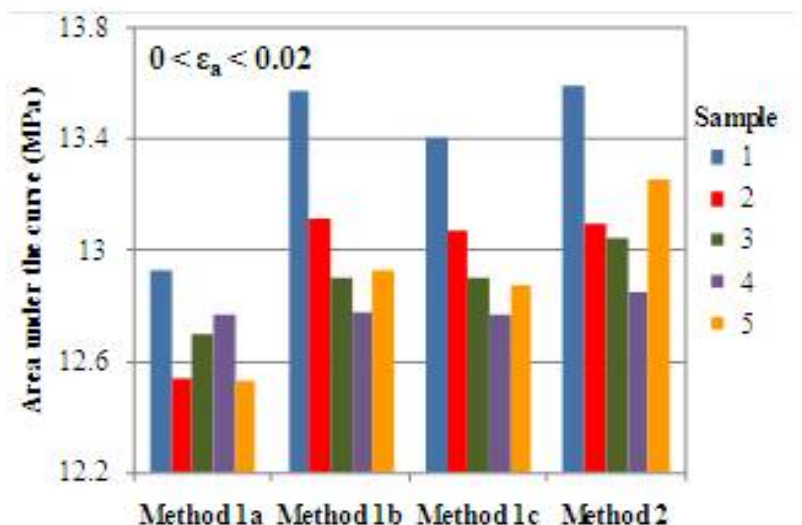

Fig. 4 Area under cyclic curves evaluated with the four methods. 
Table 3 Differences between the areas under the curves obtained using the same method on all specimens (MPa).

\begin{tabular}{lllll}
\hline $0<\varepsilon_{a}<0.02$ & \multicolumn{1}{c}{ Method 1a } & Method 1b & Method 1c & Method 2 \\
\hline Maximum & 0.412 & 0.801 & 0.642 & 0.741 \\
Mean & 0.211 & 0.325 & 0.267 & 0.316 \\
Minimum & 0.023 & 0.037 & 0.056 & 0.090 \\
\hline
\end{tabular}

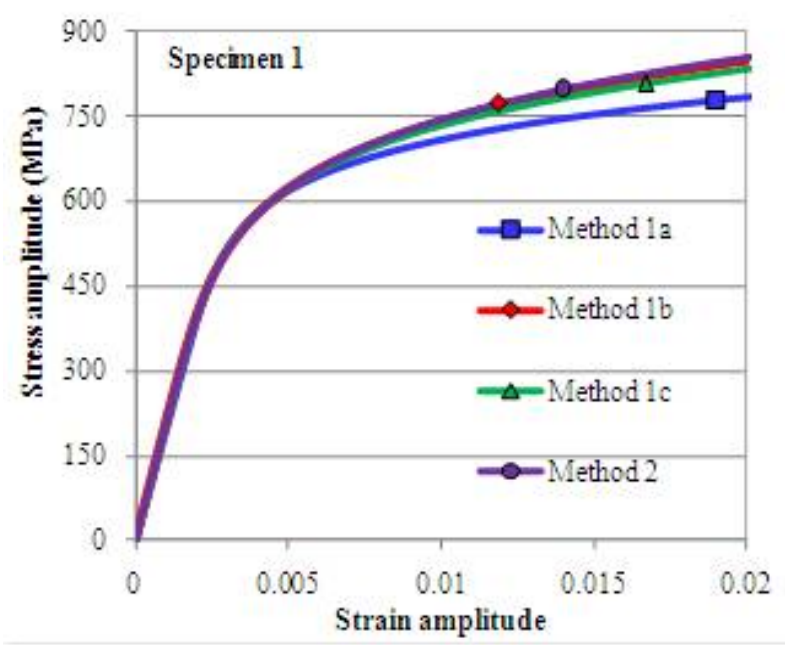

Fig. 5 Cyclic curves evaluated with different methods on sample 1.

among the cyclic stress-strain curves calculated applying the different techniques on a single specimen. The situation is similar for all samples. As it can be observed, the differences increase with $\varepsilon_{a}$. Moreover, method 2 always gives curves with the highest values, while the lowest values are obtained with method 1a.

If the Euclidian norm of the residual vectors between the experimental data and the correspondent calculated values is considered, it is clear that, for the way used to obtain it, the best approximation is associated to method 2 .

A way to enhance the differences is to evaluate the value of $\sigma_{a}$ corresponding to $\varepsilon_{a}=0.02$ (Fig. 6). The obtained values are significantly different, especially using the procedure 1a. This is probably due to lower number of experimental values used in calculating the Young's modulus (about 20 against 100 or more associated to procedures $1 \mathrm{~b}$ and $1 \mathrm{c}$ ), with an higher sensitivity of the result to the specific values acquired. The differences among the curves calculated using the four methodologies are comparable to those described in Section 4.3 and attributable to the material behaviour. Table 4 presents the average and the extreme values of the differences among the areas under the curves.

\subsection{Mean Curves}

To estimate a mean behaviour, it is possible to use the cyclic curve obtained by averaging arithmetically the parameters calculated in the five tests. If the number of the recorded data for each test is the same, an alternative way to obtain a description of the mean material response is to gather all the experimental data acquired in only one set and to carry out the following elaborations on this set. This last procedure is preferable, since in this way the best interpretation of the whole set of acquired values is obtained.

Table 5 shows the values of the coefficients $E, n^{\prime}, K^{\prime}$ evaluated both by arithmetic average and by the elaboration of the complete data set. Fig. 7 shows the ratio between the mean material parameters evaluated with the two procedures; in this case the observed

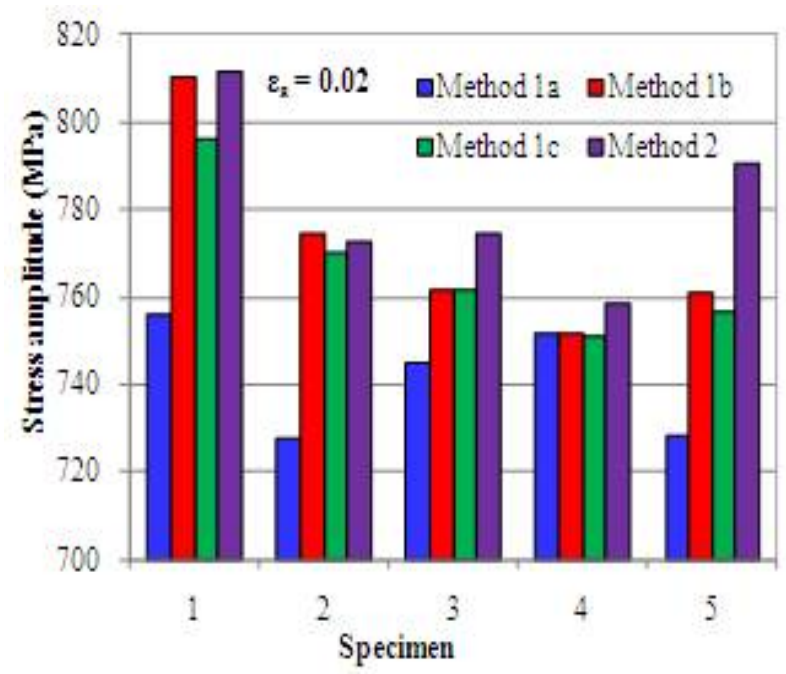

Fig. 6 Values of stress amplitude for $\varepsilon_{a}$ equal to 0.02 .

Table 4 Differences between the areas under the curves obtained with the set of methods on a single specimen (MPa).

\begin{tabular}{llllll}
\hline $0<\varepsilon_{a}<0.02$ & & & & & \\
\hline Sample & 1 & 2 & 3 & 4 & 5 \\
\hline Maximum & 0.684 & 0.589 & 0.364 & 0.091 & 0.771 \\
Mean & 0.372 & 0.300 & 0.183 & 0.049 & 0.395 \\
Minimum & 0.021 & 0.019 & 0.003 & 0.006 & 0.050 \\
\hline
\end{tabular}


Table 5 Average values of the coefficients of the cyclic curve.

\begin{tabular}{llll}
\hline & & $\begin{array}{l}\text { Average of } \\
\text { coefficients }\end{array}$ & $\begin{array}{l}\text { All data } \\
\text { elaboration }\end{array}$ \\
\hline \multirow{3}{*}{ Method 1a } & $E(\mathrm{GPa})$ & 202.0 & 207.0 \\
& $K^{\prime}(\mathrm{MPa})$ & $1,236.2$ & $1,248.9$ \\
& $n^{\prime}$ & 0.1136 & 0.118 \\
Method 1b & $E(\mathrm{GPa})$ & 211.1 & 213.0 \\
& $K^{\prime}(\mathrm{MPa})$ & $1,439.9$ & $1,436.3$ \\
& $n^{\prime}$ & 0.1389 & 0.1398 \\
Method 1c & $E(\mathrm{GPa})$ & 210.5 & 213.8 \\
& $K^{\prime}(\mathrm{MPa})$ & $1,408.5$ & 1,464 \\
& $n^{\prime}$ & 0.1356 & 0.1429 \\
Method 2 & $E(\mathrm{GPa})$ & 215.7 & 209.7 \\
& $K^{\prime}(\mathrm{MPa})$ & $1,529.2$ & $1,377.2$ \\
& $n^{\prime}$ & 0.1463 & 0.1323 \\
\hline
\end{tabular}

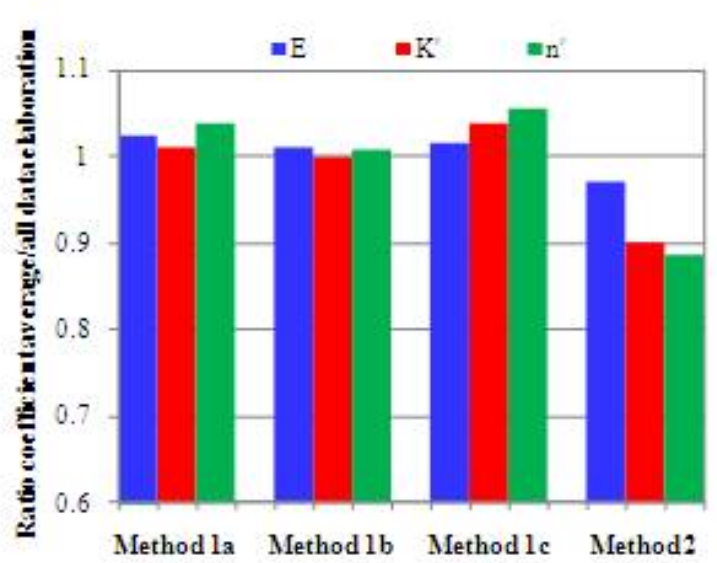

Fig. 7 Ratio between the mean material parameters evaluated with the two procedures.

differences are minimal. The corresponding cyclic curves, calculated with the four procedures, do not deviate from the behaviour described above, even if they show some small variations in their relative position. This conclusion is also confirmed by the results of the analysis of the areas under the curves, as shown in Fig. 8 and Table 6.

\section{Conclusions}

Four different methodologies were reviewed and used to evaluate the parameters useful to analytically modelling the cyclic stress - strain curve. The various techniques were applied to data obtained from tests carried out on X22CrMoV12-1 stainless steel specimens, and the resulting differences were analyzed and quantified. Young's modulus evaluation has a

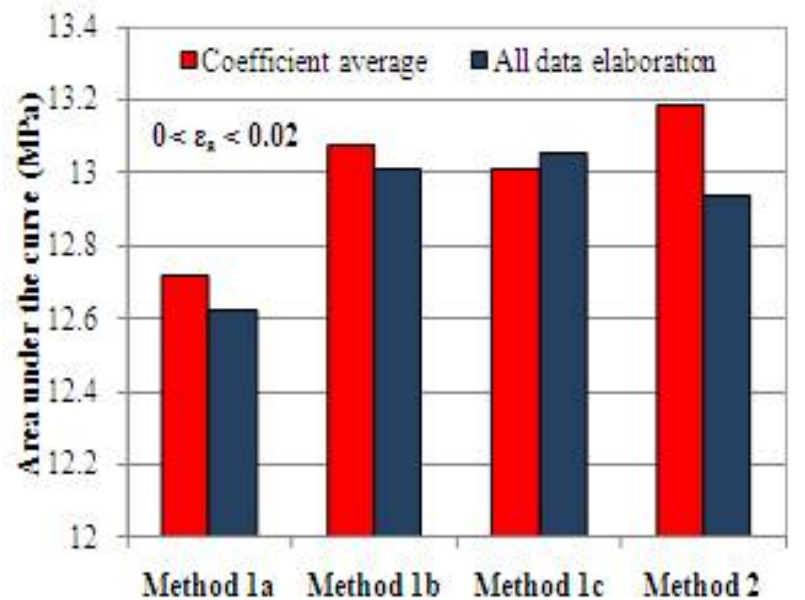

Fig. 8 Average values of the area under the cyclic curve.

Table 6 Differences between the areas under the mean curves evaluated with the set of methods (MPa).

\begin{tabular}{lll}
\hline & $\begin{array}{l}\text { Average of } \\
\text { coefficients }\end{array}$ & $\begin{array}{l}\text { All data } \\
\text { elaboration }\end{array}$ \\
\hline Maximum & 0.489 & 0.442 \\
Mean & 0.256 & 0.236 \\
Minimum & 0.067 & 0.047 \\
\hline
\end{tabular}

great influence on the obtained results: a correctly assessment of this parameter is necessary to reliable represent the behaviour of the material. To reach this goal, the best method is the 1c. The method 1a is influenced by the number of cycles in elastic field present in a block and it could provide not enough accurate results if this number is low. Method $1 \mathrm{~b}$ frequently shows different results as the portion of the hysteresis loop considered changes.

The four methodologies allow building cyclic curves which mainly differ in the plastic field. Applying these procedures to a single specimen the results are more dispersed than those obtained using a single method on a set of nominally identical samples. However, the differences are of the same order of magnitude, as quantified by the values of area under the curves. An average behaviour was evaluated considering the total set of data obtained from experimental tests. In order to assess the results, it is important to consider the strain range of interest; it is clear that increasing the value of strain amplitude results in a greater scatter of the curves, because they were built from experimental data in which the maximum strain is equal to $1 \%$. In this field 
of strain, no significant differences among results were observed.

The choice of the most suitable method is related to both the strain range of interest and the purpose for which the results will be used. The method 2 provides surely the best correspondence to the experimental data, but its use is only appropriate to describe the cyclic curve, because the values of the three parameters obtained should not be used separately. If, on the other hand, it is useful to have a single value of the modulus of elasticity to use also for other purpose, the use of methodology $1 \mathrm{c}$ is more appropriate.

\section{References}

[1] Stephens, R. I., Fatemi, A., Stephens, R. R., and Fuchs, H. O. 2001. Metal Fatigue in Engineering. 2nd edition. New York: John Wiley \& Sons.

[2] Milella, P. P. 2013. Fatigue and Corrosion in Metals. Milan: Springer Verlang Italia.

[3] Mostaghel, N., and Byrd, R. A. 2002. "Inversion of Ramberg-Osgood Equation and Description of Hysteresis Loops." Journal of Non-Linear Mechanics 37 (8): 1319-35.

[4] Lopez, Z., and Fatemi, A. 2012. "A Method of Predicting Cyclic Stress-Strain Curve from Tensile Properties for Steels." Materials Science \& Engineering A 556: 540-50.

[5] Zhang, Z., Li, J., Sun, Q., Qiao, Y., and Li, C. 2009. "Two Parameters Describing Cyclic Hardening/Softening Behaviors of Metallic Materials." Journal of Materials Engineering and Performance 18 (3): 237-44.

[6] Zhang, Z., Qiao, Y., Sun, Q., Li, C., and Li, J. 2009. "Theoretical Estimation to the Cyclic Strength Coefficient and the Cyclic Strain-Hardening Exponent for Metallic Materials: Preliminary Study." Journal of Materials Engineering and Performance 18 (3): 245-54.
[7] Tomasella, A., El Dsoki, C., Hanselka, H., and Kaufmann, H. 2011. "A Computational Estimation of Cyclic Material Properties Using Artificial Neural Networks.” Procedia Engineering 10: 439-45.

[8] Ghajar, R., Naserifar, N., Sadati, H., and Alizadeh, K. J. 2011. “A Neural Network Approach for Predicting Steel Properties Characterizing Cyclic Ramberg-Osgood Equation." Fatigue \& Fracture of Engineering Materials \& Structures 34 (7): 534-44.

[9] Dowling, N. E. 1993. Mechanical Behavior of Materials. Englewood Cliffs: Prentice-Hall.

[10] Lukas, P., and Klesnil, M. 1973. "Cyclic Plastic Reaction and Fatigue Process in Metals." Kovove Materialy 11: 340-57.

[11] Raman, S. G. S., and Radhakrishnan, V. M. 2002. "On Cyclic Stress-Strain Behaviour and Low Cycle Fatigue Life." Materials and Design 23 (3): 249-54.

[12] Morrow, J. D. 1965. "Cyclic Plastic Energy and Fatigue of Metals." In Internal Friction and Cyclic Plasticity, ASTM STP 378, edited by Lazan, B. J. Philadelphia: ASTM International.

[13] Landgraf, R. W., Morrow, J. D., and Endo, T. 1969. "Determination of the Cyclic Stress Strain Curve." Journal of Materials 4 (1): 176-88.

[14] Pickard, A. C., and Knott, J. F. 1987. "Effects of Testing Method on Cyclic Hardening Behavior in Face-Centered-Cubic Alloys." In Low Cycle Fatigue, ASTM STP 942, edited by Solomon, H. D., Halford, G. R., Kaisand, L. R., and Leis, B. N. Philadelphia: ASTM International.

[15] Schoeler, K., and Christ, H. J. 2001. "Influence of Prestraining on Cyclic Deformation Behaviour and Microstructure of a Single-Phase Ni-Base Superalloy." Int. Journal of Fatigue 23 (9): 767-75.

[16] Hales, R., Holdsworth, S. R., O’Donnell, M. P., Perrin, I. J., and Skelton, R. P. 2002. "A Code of Practice for the Determination of Cyclic Stress-Strain Data." Materials at High Temperatures 19 (4): 165-85. 